\title{
ICTs for Assessment and Intervention on Cultivation of Empathy
}

\author{
http://dx.doi.org/10.3991/ijet.v10i5.4731 \\ Athanasios S. Drigas, and Chara Papoutsi \\ NCSR DEMOKRITOS, Institute of Informatics and Telecommunications, Net Media Lab, Athens, Greece
}

\begin{abstract}
Empathy can be defined as the ability to perceive and understand others' emotional states. Neuropsychological evidence has shown that humans empathize with each other to different degrees depending on factors such as their mood, personality, and social relationships. ICTs with the features that offered are a very important tool for the detection, the development and the cultivation of empathy in children and adults in various fields and in different ways.
\end{abstract}

Index Terms-ICTs, Empathy, Autism, Bullying, Empathic Agents, Virtual Environments, Health, Games, Robots, Social Networks, Neural Mechanisms

\section{INTRODUCTION}

Rapid developments in information technology have dramatically changed the living conditions for many people during the past decades. ICTs stand for information and communication technologies and are defined, for the purposes of this primer, as a "diverse set of technological tools and resources used to communicate, and to create, disseminate, store, and manage information. These technologies include computers, the Internet, broadcasting technologies (radio and television), and telephony [1]. ICTs have been widely studied in a large number of fields as well as being a subject of study in its own right. In recent years there has been a groundswell of interest in how computers can best be harnessed in order to detect and foster empathy and to achieve empathic interactions between computers and humans.

The original German word Einfühlung, of which the English "empathy" is Titchener's translation, literally means "feeling into". Einfühlung was thought to result from a process where observers project themselves into the objects they perceive [2]. Many researchers have engaged with empathy and its bases. Empathy has been defined as "the ability to understand others' emotions and/or perspectives and, often, to resonate with others' emotional states," or as "an affective response that is identical, or very similar, to what the other person is feeling or might be expected to feel given the context: a response stemming from an understanding of another's emotional state or condition" [3]. Empathy requires both the ability to share the emotional experience of the other person (affective component) and an understanding of the other person's experience (cognitive component) [4]. Empathy can also be considered to be equivalent to conviviality that allows individuals to identify with each other thereby experiencing each other's feelings, thoughts, and attitudes and hence is deemed a central concept to design a community [5].

Understanding of the significant role of emotions and empathy, great progress has been observed in the design of ICT software programs in order to support and induce the expression of empathy in children and in adults too. For that reason applications, software, virtual environments, games, empathic pedagogical agents have been developed to measure, detect and promote empathy. These applications and software have been expanded in many fields such us : ICTs - empathy and autism, ICTs - empathy and bulling, ICTs - empathy and robots, ICTs - empathy and empathic pedagogical agents, ICTs - empathy and health, ICTs - empathy and virtual environments, ICTs empathy and games, ICTs - empathy and social networks and ICTs - empathy and neural mechanisms.

In this paper we present an overview of the most representative studies which deal with this important phenomenon of empathy. The studies chosen will be classified according to the areas of needs they serve.

\section{EMPathy In VARious Pedagogical AND Other DOMAINS}

\section{A. Empathy and Autism (ASC)}

Golan et al., (2010) created 'The Transporters', a children's animation series, based around eight characters who are vehicles that move according to rule-based motion. Such vehicles, it was hoped, would readily attract the attention of young children with ASC, both highfunctioning children-who have developed age appropriate language and cognitive abilities, and low-functioning children, who have significant learning difficulties. Onto these vehicles grafted real-life faces of actors showing emotions, and contextualized them in entertaining social interactions between the toy vehicles. The aim was to explore whether creating an autism-friendly context of predictable mechanical motion could introduce facial expressions of emotion that could then be learned more easily than is possible in the real world [6], [7].

Blocher et al., (2002) presented an interactive computer system 'Affective Social Quest' aimed at helping autistic children learn how to recognize emotional expressions. The system illustrates emotional situations and then cues the child to select which stuffed "dwarf" doll most closely matches the portrayed emotion. The dwarfs provide a wireless, playful haptic interface that can also be used by multiple players. The goal of ASQ is to provide an engaging environment to help children - specifically autistic children -learn to recognize social displays of affect [8].

\section{B. Empathy and Bullying}

One of the most widely used software tools is the VICTEC project (Virtual ICT with Empathic Characters), a European framework $\mathrm{V}$ project which was carried out between 2002-2005. The project considered the applica- 
tion of 3D animated synthetic characters and emergent narrative to create improvised dramas to address bullying problems for children aged 8-12 in the UK, Germany and Portugal. One of the main aims of the project was to develop synthetic characters that could, through their appearances, behaviours and features allow the user to build empathic relations with them. The project's pedagogical objectives were met through the design and implementation of interactive, episodic and emergent virtual dramas where children were exposed to bullying scenarios in a safe school-based Virtual Learning Environment (VLE). The FearNot! application software (Fun with Empathic Agents to Achieve Novel Outcomes in Teaching) developed during the project aimed to enable children to explore physical and relational bullying issues and coping strategies through empathic interactions with synthetic characters. In order to achieve these objectives, FearNot! provides children with various scenarios about bullying behaviour, that promote engagement and believability with synthetic characters in a social interaction [9], [10]. To build empathy into FearNot! they have considered the following components: agent's architecture; the characters' embodiment; the environment itself and emotionally charged situations [11].

The ECIRCUS project is a three year project (European framework VI project) which started in March 2006. This project intends to further develop the technology from the VICTEC project and carry out on a number of large-scale psychological evaluations in schools using both the FearNot! software and the purpose built ORIENT software to be developed in the course of the project. ECIRCUS aims to develop novel conceptual and innovative technologies to support social and emotional learning through role-play and affective engagement for Personal, Health and Social Education involving complex social situations. The main objective of this project is to enhance learning through the use of a new kind of interactive 3D environment that explores virtual play and improvisational drama with synthetic characters. The project will target PHSE in which empathy and emotional engagements are key factors, using the hard problems of bullying and refugee integration in schools as exemplars [9].

\section{Empathy and Empathic Pedagogical Agents}

Chatzara et al. designed an agent, which named Sofia who is able to express emotions by portray facial expressions that are related with learning. Sofia's goal is to communicate emotionally with the user in a pedagogical manner. Caring agent may not only increase the interaction between students and the learning system, but also have positive effects on students' emotions and engage them in learning [12].

Lim et al., (2005) introduced 'The Empathic Tour Guide System' a context-aware mobile system, including an 'intelligent empathic guide with attitude', offering the user a seamless, temporally and spatially dependent, multi-modal interaction interface. The phrase 'empathic guide with attitude' means a guide that does not only show emotions during interaction, but at the same time try to invoke empathy in the user. It will consist of two virtual agents each possessing a contrasting personality, presenting users with different versions of the story of the same event or place. An Emergent Empathic Model with Personality is proposed as a mechanism for action selection and affec- tive processing. The system will mould to the behavior of the users and facilitate their movement, applying storytelling techniques which link the memory and interests of the guide as well as the visitor to the spatial location so that stories are relevant to what can be immediately seen, creating personalised communication. Multisensory systems will be integrated with the PDA, adopting wireless technology. Detection of the user's current physical position will be performed by a Global Positioning System [13].

Prendiger et al., (2005) developed 'the Empathic Companion', an animated interface agent that accompanies the user in the setting of a virtual job interview. This interface application takes physiological data (skin conductance and electromyography) of a user in realtime, interprets them as emotions, and addresses the user's affective states in the form of empathic feedback. The Empathic Companion is conceived as an educational agent that supports job seekers preparing for a job interview [14].

Woolf et al., (2010) developed 'Wayang Outpost' ("Wayang") who is an intelligent tutor that helps students prepare for standardized tests that assess general mathematic skills. 'Wayang' includes a wide range of metacognitive and affective support, delivered by learning companions; agents designed to act like peers who care about a student's progress, and offer affective and empathic support and advice on how to improve student learning strategies [15].

Kim (2005) presented empathetic, pedagogical agents 'PALs' that tried to enhance learner interest and selfefficacy through empathetic responses and interaction. The 'PALs' were developed using Poser 5, a 3Dimage/animation-design tool, and Mimic Pro 2, a voice/affect-editing tool. The animation files created in Poser 5 were converted to Macromedia Flash movies for compression and were later integrated into the instructional intervention. The 'PALs', Chris, (one version male, one female) were designed to look about twenty years old, casually dressed and speaking informally, sometimes using slang. Male and female voices of college students were recorded [16].

\section{Empathy and Neural Mechanisms}

Jackson et al. (2006) used a series of 96 digital color pictures showing right hands and right feet of people in painful and non-painful situations. All situations depicted familiar events that can happen in everyday life to people (e.g. pinching one's finger in a door, or catching one's toe under a heavy object). Various types (mechanical, thermal and pressure) and levels (neutral [no pain], low pain, medium pain, high pain) of pain inflicted to the limbs were depicted. Three different perspectives were manipulated through explicit instructions: the subject's own perspective (Self), that of a specific but unfamiliar person (Other), and that of a plastic limb (Artificial). Stimuli were presented with a computer running, the Presentation software (Neurobehavioural Systems TM) to manage the timing and presentation of the stimuli, as well as the recording responses [17].

\section{E. Empathy and Health (Mental and Physical)}

Bickmore and Picard (2005) developed an embodied exercise advisor named Laura, which attempted to build and maintain a relationship with subjects who interacted with the agent daily, for a month. Laura used a number of 
strategies that humans use to establish and maintain relationships, including social, empathetic and polite communication, talk of the relationship, humor and appropriate forms of address in order to help people improve their exercise habits [18].

Liu and Picard (2005) had subjects interact with a mobile health device and then analysed the effect it had on subject's attitudes and behavior. The mobile device was consisted of an empathetic agent who would make empathic comments when interacting with the subject in order to discuss issues relating to the user's health [19].

Lindsay et al. (2012) developed the 'KITE project'. The 'KITE project' design work examined the potential for designing a digital intervention to promote safe walking for people with dementia, i.e. walking outdoors unaccompanied by a caregiver with minimal risk of becoming lost or experiences of anxiety. Specifically, the process aims to foster an empathic relationship between designers, people with dementia and caregivers by demanding close, respectful contact. The approach focuses on trying to develop a holistic understanding of people with dementia's dayto-day lives and acknowledges that there is likely to be a wide gulf between designers ${ }^{\text {ee }}$ and people with dementia's life experiences [20].

Stacy C. Marsella (2002) introduced an Interactive Pedagogical Drama (IPD), 'Carmen's Bright IDEAS', which is designed to improve the social problem solving skills of mothers of pediatric cancer patients. In an interactive pedagogical drama, a learner (human user) interacts with believable characters in a believable story that the learner empathizes with. In particular, the characters may be facing and resolving overwhelming, emotionally charged difficulties similar to the learner's. The learner's identification with the characters and the believability of their problems are central to the goals of having the learner fully interact with the drama, accept the efficacy of the skills being employed in it and subsequently apply those skills in her own life [21].

Newall and Hall (2005) described the use of empathic agents in the development of an interactive pedagogical drama for the recognition and treatment of depression in adolescents. Empathic interaction by social agents can provide a useful forum for those suffering depressive episodes to obtain treatment. The user interacts with believable characters in a believable story where the characters participate in a storyline the user can empathise with. The characters in the story face and resolve problems similar to those the user is facing, so they can empathise with the characters in the drama and apply the problem solutions found to their own circumstance [22].

\section{ICT CLASSIFICATIONS OF APPLICATIONS FOR EMPATHY}

\section{A. Empathy and Social Networks}

Ortega-Ruiz et al., (2012) designed 'ConRed' a program, which addresses cyberbullying and other emerging problems linked with the use of the internet and seeks to promote a positive use of this new environment. The main aims of the 'ConRed' program are a) to improve perceived control over information on the internet, b) to reduce the time dedicated to digital device usage, and c) to prevent and reduce cyberbullying. To achieve these objectives especially the last one an attempt was made to improve social skills and cultivate empathy through virtual scenarios [23].

Facebook is a sophisticated multimedia online social networking where people can gather, socialize and through interactions emotions and empathy can be emerged. Facebook is uniquely able to see a "macro" view of empathy across the network, and therefore can propose "interventions" that will influence social networks and communities in a number of ways [24].

Pfeil et al., (2007) (www.seniornet.org) presented 'Senior Net', an online community for older people. This online community for older people will display a high degree of emotional support and trust especially when suffering from illness. The emotional support of the online community is seen as a helpful addition to the offline recovery program [25].

\section{B. Empathy and Virtual Environments}

Nimis project (Networked Interactive Media in Schools) is an EU funded international project, produced a highly interactive infant classroom in which computers and interactive boards embedded thoughtfully in classroom environments enabled frequent high quality interaction though increased collaboration. Video analysis revealed higher levels of positive emotion and engagement than in the normal classroom. Emotional scaffolding was usually greater between adults and students than between students. Empathy is central to ensuring the quality of human communication and personal development [26], [27].

The ICT and Whole Child Project, funded by the Nuffield Foundation, developed emotional scales and compared a normal classroom with an ICT embedded classroom, over two years. Affective issues were revealed to be at the heart of learning and positive emotion was generated in the computer classroom where teachers and children continually collaborated around the machines. Achievements at the end of two years were higher in the computer embedded classrooms, as was self-esteem [26], [28].

McQuiggan et al., (2006) presented 'CARE', a datadriven affective architecture and methodology for learning models of empathy by observing human-human social interactions. First, in CARE training sessions, one trainer directs synthetic agents to perform a sequence of tasks while another trainer manipulates companion agents' affective states to produce empathetic behaviors (spoken language, gesture, posture). Treasure Hunt is a prototype virtual environment featuring a synthetic agent controlled by the user and a companion agent whose empathetic behaviors are controlled by CARE. The user navigates the $3 \mathrm{D}$ virtual world in search of hidden (and some not- sohidden) treasures. Each treasure box is labeled with the value of its contents, representing points the user obtains when collecting the associated treasure. Throughout the users' quest for treasure, the companion agent follows along and expresses empathetic behaviors as appropriate situations arise in the users' experiences [29], [30].

McQuiggan et al., (2008) and Sabourin et al., (2011) presented 'CRYSTAL ISLAND', a virtual environment which is designed to teach the domains of microbiology and genetics to middle school students. It features a science mystery set on a recently discovered volcanic island where a research station has been established to study the unique flora and fauna. The user plays the protagonist, Alex, attempting to discover the genetic makeup of the 
chickens whose eggs are carrying an unidentified infectious disease at the research station. The story opens by introducing the student to the island and the members of the research team for which her father serves as the lead scientist. The empathetic model is developed through the interactions between the subjects and the virtual characters [29], [31].

Hall et al., (2011) introduced the 'eCute project'. The 7 th Framework EU project 'Education for Cultural Understanding Technology Enhanced' (eCute) aims to develop cultural understanding by providing immersive virtual role play with intelligent interactive graphical characters embodying models of culturally-specific behaviour and interaction. Helping children and young people to develop empathy for people from other cultures is therefore an ever pressing pedagogical imperative. interactive graphical characters embodying models of culturally-specific behaviour and interaction. Targeted at two specific age groups, children aged 9-11 and young adults aged 18-25, 'eCute' sets out to design and build two cultural Virtual Learning Environments (VLEs) based on virtual dramas and to evaluate these with stakeholder teacher and learner groups to demonstrate learning efficacy [32].

\section{Empathy and Games}

Belman et al., (2010) presented three games to cultivate empathy. The games are: 'Peacemaker', 'Hush' and 'Layoff'. In 'Peacemaker' the player inhabits the role of either the Israeli Prime Minister or Palestinian President during a particularly volatile period of the PalestinianIsraeli conflict. Whichever role the player chooses, the goal is to create conditions in which a "two-state solution" to the conflict becomes viable. There are a wide variety of actions to choose from, some hawkish, some conciliatory, some unilateral, and some that require cooperation with groups on the other side of the conflict. The game encourages empathy in several ways [33].

'Hush' begins with a screen prompting us to take the perspective of the player character, who is a Rwandan Tutsi mother hiding in a shack with her baby during the genocide of 1994. Against a background of haunting music, this message appears: Rwanda, 1994: The Hutu are coming, Liliane. Hide your child. If you falter in your lullaby, he will grow restless. The soldier will hear him, and he will come for you. By addressing the player as "Liliane," the game encourages players to forego the emotional distance that usually separates them from what happens on screen. This can be regarded as a kind of empathy induction, in response to which we may be more likely to inhabit, explore, and identify with Liliane's experience [33].

'Layoff' is designed to elicit empathy in players towards characters in the game. In Layoff, one plays as "corporate management," tasked with cutting jobs during the financial crisis. When players match sets of three or more workers, they fall off the bottom end of the board into an "unemployment office." The game, however, is designed to challenge this perspective, to contend with the idea that a worker is only a "part." Each worker has a detailed personal biography that pops up when their tile is selected. In Layoff a bond of empathy is created not between the player and player character (who in Layoff, represents management), but rather between the player and non-player characters (i.e., the workers who are being laid off) [33].

Gordon et al., (2011) studied the implementation of 'Participatory Chinatown' a 3D, multiplayer game designed to be played in the shared physical space of a master planning meeting in Boston's Chinatown neighborhood. 'Participatory Chinatown' demonstrates that roleplay can engage players in local issues and motivate them to engage with each other and with their community. When playing the game, participants empathize with the needs and desires of a character and make decisions accordingly [34].

Bachen et al., (2012) examined 'REAL LIVES', a game that allows learners to live simulated lives in other countries and to develop global empathy, identification, and interest in learning about other countries. Students can vicariously experience what life could be like for a male or female in another country, including education, employment, marriage, having children, confronting diseases and natural disasters, and so on. The software uses realworld data to determine the probability of events that are likely to happen in the character's life in their particular birth country. 'REAL LIVES' contains elements that could foster the cognitive, affective, and communicative components of global empathy [35].

\section{Empathy and Robots}

Canãmero (2002) created 'Feelix', a 70cm-tall "humanoid" robot (Figure 8.1) built from commercial LEGO Mindstorms ${ }^{\mathrm{TM}}$ robotic construction kits. 'Feelix' expresses emotions by means of its face. To interact with the robot, people sit or stand in front of it. Since the interaction wanted to be as natural as possible, the feet seemed the best location for tactile stimulation, as they are protruding and easy to touch; thus a binary touch sensor was attached underneath each foot. 'Feelix's' face has four degrees of freedom (DoF) controlled by five motors, and makes different emotional expressions by means of two eyebrows (1DoF) and two lips (3 DoF). The robot is controlled on-board by two LEGO MindstormsRCX ${ }^{\mathrm{TM}_{\mathrm{M}}}$ computers3, which communicate via infrared messages [36].

Scheeff et al., (2002) built 'Sparky' which is a small robot about $60 \mathrm{~cm}$ long, $50 \mathrm{~cm}$ high and $35 \mathrm{~cm}$ wide. It has an expressive face, a movable head on a long neck, a set of moving plates on its back and wheels for translating around the room. Even though high-level commands are sending to the robot from a remote location, 'Sparky' seems autonomous to those around it. The robot uses gesture, motion and sound to be social with humans in the immediate vicinity [37].

Breazeal et al., (2000) designed and built a robot named 'Kismet' to provide emotional feedback to the caretaker through facial expressions. It consists of an active stereo vision system embellished with facial features for emotive expression. Currently, these facial features include eyebrows (each with two degrees of freedom: lift and arch), ears (each with two degrees of freedom: lift and rotate), eyelids (each with one degree of freedom: open/close), and a mouth (with one degree of freedom: open/close). The robot is able to show expressions analogous to anger, fatigue, fear, disgust, excitement, happiness, interest, sadness, and surprise which are easily interpreted by an untrained human observer [38]. 
Leite et al., (2012) developed 'iCat Robot', a social robot capable of recognising and responding empathically to some of the children's affective states while playing a chess game. The goal was to see how children perceive and interact with an empathic social robot in their school environment while playing the game. The empathic model includes an affect detector that allows the robot to infer the valence of the feeling experienced by the children. The affect detector, based on Support Vector Machines (SVMs), processes in real-time behavioural and contextual features, providing as output a probability value for each of the following valence conditions: positive, neutral and negative [39].

\section{CONCLUSIONS}

The scope of this study was to examine the most representative studies which exploiting ICT and reveals how helpful ICTs can be with the important phenomenon of empathy. The use of ICT has played a major part in assessment and intervention of empathy in different domains in children and in adults too. However, it is essential for increased research in this area so as to employ more ICTs strategies to accomplish more in the detection and cultivation of empathy. There is still significant work to be done in investigating the effects of embedding empathy into health technology systems that interact with people in order to help people especially those ones who suffer from mental diseases. Furthermore aspects of the relations between users and empathetic characters such as proximity, situation must also be looked at as well as emotional body and facial expression. Investigation must be done in order to explore how particular design features and strategies are associated with eliciting different kinds of empathy, and to better understand whether and how "empathetic play" influences players' attitudes and behaviors. Because empathy is a natural extension of the appraisal process and appears continuously in human-human interaction, it is important to endow virtual agents with the capability to respond with the parallel and reactive empathetic expressions that are most appropriate for the user, her situation and her affective state.

Empathy could be analyzed in a long-term study, achieving greater accuracy by studying more messages and by studying the evolution of the community. It would be also interesting to investigate more of empathy as it occurs in online communities and in synchronous chat rooms. However, an important area of future work is to design games to foster empathy or apply the existing ones in larger samples. With that way software such us VICTEC can be technically improved so as to expand its' content to problems of refugee integration in schools, and to develop and implement a suitable longitudinal evaluation approach to assess the long-term effects of the software on stable empathic styles and bullying roles. Further research is also needed to verify if the results can be extrapolated for designing empathic social robots in different application domains and for other target users such as the elderly.

The current study is encouraging and there is a general consensus that ICTs do play a significant role in ensuring and enhancing empathy to achieve more in bulling, in learning process, in health, in human - computer interaction, in virtual environments etc.

\section{REFERENCES}

[1] Tinio, V. L. (2003). ICT in Education. United Nations Development Programme-Asia Pacific Development Information Programme.

[2] Preston, S. D., \& De Waal, F. (2002). Empathy: Its ultimate and proximate bases. Behavioral and brain sciences, 25(01), 1-20.

[3] Eisenberg N, Eggum ND, Di Giunta L (2010) Empathy-related responding: associations with prosocial behavior, aggression, and intergroup relations. Soc Issues Policy Rev 4(1):143-180. http://dx.doi.org/10.1111/j.1751-2409.2010.01020.x

[4] Decety, J., \& Jackson, P. L. (2004). The functional architecture of human empathy. Behavioral and cognitive neuroscience reviews, 3(2), 71-100. http://dx.doi.org/10.1177/1534582304267187

[5] Caire P (2009) Designing convivial digital cities: a social intelligence design approach. AI Soc 24(1):97-114. http://dx.doi.org/10.1007/s00146-009-0201-x

[6] Golan, O., Ashwin, E., Granader, Y., McClintock, S., Day, K., Leggett, V., \& Baron-Cohen, S. (2010). Enhancing emotion recognition in children with autism spectrum conditions: An intervention using animated vehicles with real emotional faces. Journal of autism and developmental disorders, 40(3), 269-279. http://dx.doi.org/10.1007/s10803-009-0862-9

[7] Boucenna, S., Narzisi, A., Tilmont, E., Muratori, F., Pioggia, G.,Cohen, D., \& Chetouani, M. (2014). Interactive technologies for autistic children: A review. Cognitive Computation, 6(4), 722740. http://dx.doi.org/10.1007/s12559-014-9276-x

[8] Blocher, K., \& Picard, R. W. (2002). Affective social quest. In Socially Intelligent Agents (pp. 133-140). Springer US. http://dx.doi.org/10.1007/0-306-47373-9_16

[9] Louchart, S., Aylett, R., Hall, L., Woods, S., \& Paiva, A. FearNot! developing social immersion in the VICTEC and ECIRCUS project.

[10] Zoll, C., Enz, S., Schaub, H., Aylett, R., \& Paiva, A. (2006, April) Fighting bullying with the help of autonomous agents in a virtual school environment. In 7th International Conference on Cognitive Modelling.

[11] Paiva, A., Dias, J., Sobral, D., Aylett, R., Woods, S., Hall, L., \& Zoll, C. (2005). Learning by feeling: Evoking empathy with synthetic characters. Applied Artificial Intelligence, 19(3-4), 235-266. http://dx.doi.org/10.1080/08839510590910165

[12] Chatzara, K., Karagiannidis, C., \& Stamatis, D. Study on intelligent emotional agents for learning.

[13] Lim, M. Y., Aylett, R., \& Jones, C. M. (2005, April). Empathic interaction with a virtual guide. In Proceeding of the Joint Symposium on Virtual Social Agents, AISB (Vol. 5, pp. 122-129).

[14] Prendinger, H., \& Ishizuka, M. (2005). The empathic companion: A character-based interface that addresses users'affective states. Applied Artificial Intelligence, 19(3-4), 267-285. http://dx.doi.org/10.1080/08839510590910174

[15] Woolf, B. P., Arroyo, I., Muldner, K., Burleson, W., Cooper, D. G., Dolan, R., \& Christopherson, R. M. (2010, January). The effect of motivational learning companions on low achieving students and students with disabilities. In Intelligent Tutoring Systems (pp. 327-337). Springer Berlin Heidelberg. http://dx.doi.org/10.1007/978-3-642-13388-6 37

[16] Kim, Y. (2005, July). Empathetic virtual peers enhanced learner interest and self-efficacy. In Workshop on Motivation and Affect in Educational Software, in conjunction with the 12th International Conference on Artificial Intelligence in Education (pp. 9-16).

[17] Jackson, P. L., Brunet, E., Meltzoff, A. N., \& Decety, J. (2006). Empathy examined through the neural mechanisms involved in iumagining how I feel versus how you feel pain. Neuropsychologia, 44(5), 752-761. http://dx.doi.org/10.1016/j.neuropsychologia. 2005.07.015

[18] Beale, R., \& Creed, C. (2009). Affective interaction: How emotional agents affect users. International Journal of HumanComputer Studies, 67(9), 755-776. http://dx.doi.org/10.1016/ j.ijhcs.2009.05.001

[19] Liu, K., \& Picard, R. W. (2005, April). Embedded empathy in continuous, interactive health assessment. In CHI Workshop on HCI Challenges in Health Assessment (Vol. 1, No. 2, p. 3). 
[20] Lindsay, S., Brittain, K., Jackson, D., Ladha, C., Ladha, K., \& Olivier, P. (2012, May). Empathy, participatory design and people with dementia. In Proceedings of the SIGCHI Conference on $\mathrm{Hu}$ man Factors in Computing Systems (pp. 521-530). ACM.

[21] Marsella, S. C. (2002). Pedagogical soap. In Socially Intelligent Agents (pp.141-148). Springer US. http://dx.doi.org/10.1007/0306-47373-9 17

[22] Newall, L., \& Hall, L. (2005). Using Empathic Agents to Prevent and Treat Depression in Adolescents. Virtual Social Agents, 95.

[23] Ortega-Ruiz, R., Del Rey, R., \& Casas, J. A. (2012). Knowing, building and living together on internet and social networks: The ConRed cyberbullying prevention program. International Journal of Conflict and Violence, 6(2), 302-312.

[24] Venkatanathan, J., Karapanos, E., Kostakos, V., \& Gonçalves, J. (2013, August). A network science approach to modelling and predicting empathy. In Advances in Social Networks Analysis and Mining (ASONAM), 2013 IEEE/ACM International Conference on (pp. 1395-1400). IEEE. http://dx.doi.org/10.1145/2492517. 2500295

[25] Pfeil, U., \& Zaphiris, P. (2007, April). Patterns of empathy in online communication. In Proceedings of the SIGCHI conference on Human factors in computing systems (pp. 919-928). ACM. http://dx.doi.org/10.1145/1240624.1240763

[26] Cooper, B. (2006, July). The significance of emotion and empathy in learning with MC3. In Advanced Learning Technologies, 2006. Sixth International Conference on (pp. 1204-1205). IEEE.

[27] Cooper, B., Brna, P., \& Martins, A. (2000). Effective affective in intelligent systems-building on evidence of empathy in teaching and learning. In Affective interactions (pp. 21-34). Springer Berlin Heidelberg. http://dx.doi.org/10.1007/10720296_3

[28] Cooper, B. L., \& Brna, P. (2002). Hidden curriculum, hidden feelings; emotions, relationships and learning with ICT and the whole child.

[29] McQuiggan, S. W., Robison, J. L., Phillips, R., \& Lester, J. C. (2008, May). Modeling parallel and reactive empathy in virtual agents: An inductive approach. In Proceedings of the 7th international joint conference on Autonomous agents and multiagent systems-Volume 1 (pp. 167-174). International Foundation for Autonomous Agents and Multiagent Systems.

[30] McQuiggan, S. W., \& Lester, J. C. (2006, May). Learning empathy: a data-driven framework for modeling empathetic companion agents. In Proceedings of the fifth international joint conference on Autonomous agents and multiagent systems (pp. 961-968). ACM. http://dx.doi.org/10.1145/1160633.1160806

[31] Sabourin, J., Mott, B., \& Lester, J. (2011). Computational models of affect and empathy for pedagogical virtual agents. In Standards in emotion modeling, Lorentz Center International Center for workshops in the Sciences.

[32] Hall, L., Jones, S. J., Aylett, R., André, E., Paiva, A., Hofstede, G. J., \& Nishida, T. (2011). Fostering Empathic Behaviour In Children And Young People: Interaction With Intelligent Characters Embodying Culturally Specific Behaviour In Virtual World Simulations. INTED2011 Proceedings, 2804-2814.
[33] Belman, J., \& Flanagan, M. (2010). Designing Games to Foster Empathy. International Journal of Cognitive Technology, 15(1), 11.

[34] Gordon, E., \& Schirra, S. (2011, June). Playing with empathy: digital role-playing games in public meetings. In Proceedings of the 5th International Conference on Communities and Technologies (pp. 179-185). ACM. http://dx.doi.org/10.1145/2103354. 2103378

[35] Bachen, C. M., Hernández-Ramos, P. F., \& Raphael, C. (2012). Simulating REAL LIVES: Promoting global empathy and interest in learning through simulation games. Simulation \& Gaming, 1046878111432108. http://dx.doi.org/10.1177/1046878111432108

[36] Cañamero, L. D. (2002). Playing the emotion game with feelix. In Socially intelligent agents (pp. 69-76). Springer US. http://dx.doi.org/10.1007/0-306-47373-9 8

[37] Scheeff, M., Pinto, J., Rahardja, K., Snibbe, S., \& Tow, R. (2002). Experiences with Sparky, a social robot. In Socially Intelligent Agents (pp. 173-180). Springer US. http://dx.doi.org/10.1007/0306-47373-9_21

[38] Breazeal, C., \& Scassellati, B. (2000). Infant-like social interactions between a robot and a human caregiver. Adaptive Behavior, 8(1), 49-74. http://dx.doi.org/10.1177/105971230000800104

[39] Leite, I., Castellano, G., Pereira, A., Martinho, C., \& Paiva, A. (2012, March). Modelling empathic behaviour in a robotic game companion for children: an ethnographic study in real-world settings. In Proceedings of the seventh annual ACM/IEEE international conference on Human-Robot Interaction (pp. 367-374). ACM. http://dx.doi.org/10.1145/2157689.2157811

\section{AUTHORS}

Athanasios Drigas is a Research Director at IIT, N.C.S.R. Demokritos. He is the Coordinator of Telecoms Lab and founder of Net Media Lab since 1996. From 1985 to 1999 he was the Operational manager of the Greek Academic network. He has been the Coordinator of Several International Projects, in the fields of ICTs, and eservices (e-learning, e-psychology, e-government, einclusion, e-culture etc). He has published more than 270 articles, 7 books, 25 educational CD-ROMs and several patents. He has been a member of several International committees for the design and coordination of Network and ICT activities and of international conferences and journals. Also he has accepted several distinctions for his work (articles, projects, patents) (e-mail: dr@iit.demokritos.gr).

Chara Papoutsi (MA in Applied Pedagogy) is a teacher in a primary school. She is also with NCSR DEMOKRITOS, Institute of Informatics and Telecommunications, Net Media Lab, Athens, Greece. (e-mail: xara.papoutsi@yahoo.com).

Submitted 18 May 2015. Published as resubmitted by the authors 30 October 2015. 